Document downloaded from:

http://hdl.handle.net/10251/103619

This paper must be cited as:

Park, Y.; Jeong, M.; Sang Bin Lee; Antonino-Daviu, J.; Teska, M. (2017). Influence of Blade Pass Frequency Vibrations on MCSA-based Rotor Fault Detection of Induction Motors. IEEE Transactions on Industry Applications. 53(3):2049-2058. doi:10.1109/TIA.2017.2672526

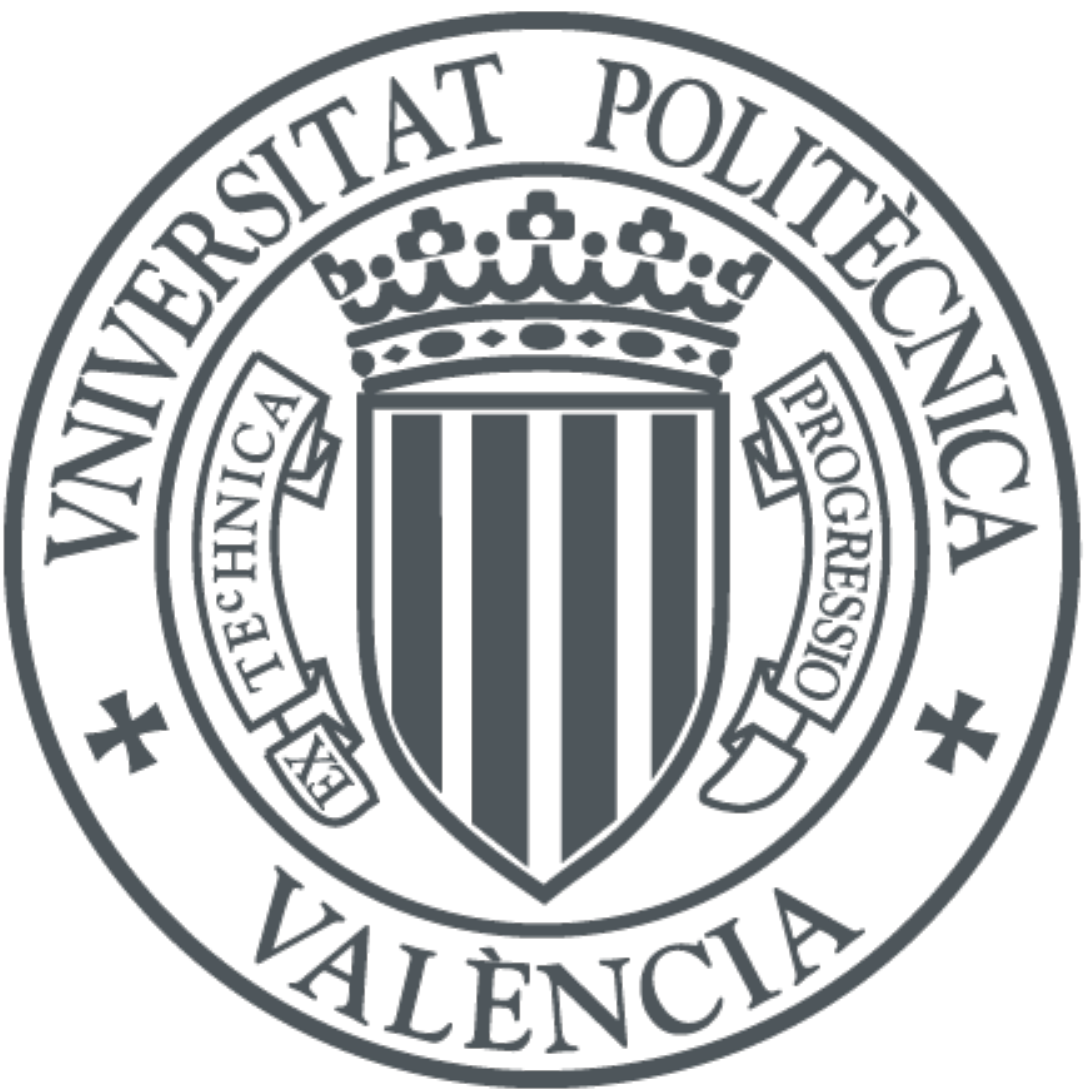

The final publication is available at

http://doi.org/10.1109/TIA.2017.2672526

Copyright Institute of Electrical and Electronics Engineers

Additional Information 


\title{
Influence of Blade Pass Frequency Vibrations on MCSA-based Rotor Fault Detection of Induction Motors
}

\author{
Yonghyun Park*, Myung Jeong ${ }^{*}$, Sang Bin Lee*, Jose Alfonso Antonino-Daviu ${ }^{* *}$, and Mike Teska ${ }^{* * *}$ \\ *Department of Electrical Engineering, \\ ${ }^{* *}$ Instituto Tecnológico de la Energía \\ Universitat, Politècnica de València, \\ Valencia, Spain \\ ${ }^{* * *}$ SKF Condition Monitoring Center, \\ Fort Collins, CO, U.S.A \\ Korea University, Seoul, Korea \\ Email: sangbinlee@,korea.ac.kr
}

inspection, which can cost up to hundreds of thousands of USD
for medium-high voltage motors, and false negative indications
can result in forced outage of the motor and process, for which
the cost can be orders of magnitude higher. False MCSA fault
indications are a major concern in the field considering the
consequences; however, there still are many cases where the
root cause of false indications cannot be explained.

Abstract-Motor current signature analysis (MCSA) has recently become widespread in industry for on-line detection of rotor cage faults in induction motors for preventing forced outages. Although it can provide low cost, remote monitoring of rotor faults, cases of false indications have been reported, where the causes of some false indications are still unknown. It is shown for the first time in this work that high-amplitude blade pass frequency (BPF) vibrations produced in pumps, fans, or compressors can cause false rotor fault indications, if the number of motor poles is an integer multiple of the number of blades. The influence of BPF vibration on MCSA based rotor fault detection is analyzed, and it is shown that the interaction between BPF vibration and rotor faults can produce false positive and negative fault indications. Alternative test methods capable of separating the influence of the BPF vibration and rotor faults are suggested for avoiding false MCSA alarms. The claims made in the paper are verified experimentally on a custom-built $380 \mathrm{~V}$ induction motor-centrifugal pump system setup.

\section{INTRODUCTION}

Predictive maintenance of induction motors based on online monitoring is the preferred maintenance strategy used in industrial facilities, as it allows efficient fault monitoring for preventing potential forced outages due to motor failures. Recently, motor current signature analysis (MCSA) is being increasingly employed in the field for detecting and preventing rotor cage failures since it can provide low cost, remote monitoring with a well-established fault threshold. Although MCSA has been proven to be successful in the field for detecting rotor faults, numerous cases and root causes of false indications have also been reported [1]-[7]. When MCSA based rotor fault detection is performed on medium-high voltage motors, there are cases where the rotor fault component is present for motors that do not show rotor damage during visual inspection. There are also cases where MCSA is unable to detect defects or faults that can be found during inspection.

The root cause of false indications has been identified to be related to the influence of the rotor structure or the load /coupling. Asymmetry in the rotor due to axial air ducts, core magnetic anisotropy/ovality, or porosity can produce a false positive indication where a fault alarm is produced in the absence of the fault [7]-[11]. Outer cage faults in double cage motors or non-adjacent broken bars can result in false negative indications where the fault is unobservable in the presence of the fault [7], [12]-[16]. Low frequency oscillations in the load due to speed reduction couplings or load variation can also produce false positive or negative indications [7], [17]-[20]. False positive indications result in unnecessary motor
The first step towards identifying false rotor fault indications for preventing unnecessary inspection or a potential forced outage is to understand what causes it. In this work, it is shown that blade pass frequency (BPF) vibrations produced in pumps, fans, or compressor loads shown in Fig. 1 can cause false indications when applying MCSA for rotor fault detection. Considering that over $60 \%$ of industrial motors are used for driving pump, fan, or compressor loads [21], it is important to understand how BPF influences MCSA. Interference due to BPF vibration is completely different from the torque oscillations produced by rotor or load rotational speed frequencies studied in [1]-[4], [16]-[20], and has not been investigated in the existing literature. The influence of BPF vibration on MCSA based rotor fault detection is analyzed in this paper, and it is shown that high-amplitude BPF vibration can produce both false positive and negative fault indications. Alternative options for reliable detection of rotor faults immune to BPF interference are devised and presented based on the analysis. The conclusions drawn from the analysis on the BPF influence and test methods are verified experimentally on a custom-built motor-centrifugal pump system.

\section{INFLUENCE OF BPF VIBRATION ON MCSA}

\section{A. Blade Pass Frequency (BPF) Vibration}

A significant percentage of medium-high voltage motors

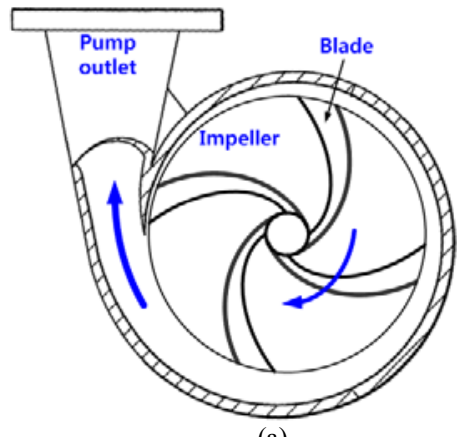

(a)

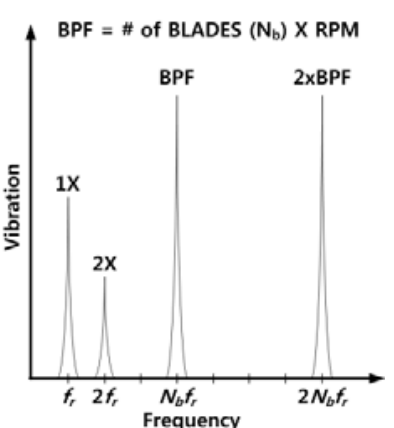

(b)
Fig. 1 (a) Centrifugal pump with a 4 blade impeller $\left(N_{b}=4\right)$, and (b) vibration spectrum for pump/fan/compressor with 4 blades/cylinders 
are used for driving fans (blowers), pumps, or compressors for creating flow for moving liquids, gases, or slurries. The rotating or reciprocating blades (vanes) or pistons employed in such equipment convert the mechanical rotating energy of the motor to increase the velocity and pressure of the fluid/gas for creating the flow [22]. The blades produce periodic vibration at frequencies that are determined depending on the speed of rotation, number of blades, and mechanical structure of the system. The structure of a centrifugal water pump with a 4 blade impeller is shown in Fig. 1(a), where the direction of the water inflow is in the axial direction into the page, and the direction of outflow is vertically upwards. As the impeller rotates, pressure impulses are produced whenever the blade (or cylinder) is hydro-dynamically loaded within the pump, fan, or compressor when it passes a discontinuity, as can be seen in Fig. 1. As a result, vibration defined as the "blade pass frequency (BPF)" vibration proportional to the product of the number of blades (or cylinders), $N_{b}$, and rotating speed of the rotor, $f_{r}$, is produced [22]-[24]. The rotor rotational speed frequency (or $1 \mathrm{X}$ frequency), $f_{r}$, and $\mathrm{BPF}, f_{v i b, B P F}$ are given by

$$
\begin{gathered}
f_{r}=2(1-s) f_{s} / P, \\
f_{v i b, B P F}=k_{1} N_{b} f_{r}
\end{gathered}
$$

where $s, f_{s}, P$, and $k_{1}$ are the slip, supply frequency, number of poles, and a positive integer, respectively.

The BPF component is inherent in pumps, fans and compressors, and observable in the frequency spectrum of acceleration, velocity, or displacement when vibration analysis is performed. BPF vibration is usually present, and does not indicate a problem unless its amplitude becomes excessive or causes an acoustic problem. The BPF vibration can increase in amplitude if defects or faults are present in the system or if structural resonance is excited. Large $f_{v i b, B P F}$ components can be observed due to problems in pumps, fans, or compressors such as non-uniform gap/clearance, disruption of flow, component wear, or resonance [21]-[22]. If the BPF vibration is excessive, it can cause accelerated wear or damage of the system components, and also induce noticeable $f_{B P F}$ components in the stator current spectrum.

It is shown in a number of resources that radial vibrations produced by motor mechanical faults result in periodic oscillations in the induced torque that modulate the stator current at the fault characteristic frequencies [25]-[26]. If the amplitude of the $f_{\text {vib,BPF }}$ is large, it can also produce fundamental frequency sidebands observable in the stator current spectrum. The influence of BPF vibration observable in the stator current with MCSA is given by

$$
f_{B P F}=f_{s} \pm k_{1} N_{b} f_{r}=f_{s} \pm 2 k_{1} N_{b}(1-s) f_{s} / P .
$$

\section{B. Interaction between BPF Vibration and Rotor Faults}

If the number of poles is an integer multiple of the number of blades, i.e. if $k_{1} N_{b} / P$ of (3) is equal to 1 and the BPF vibration is high, the influence of BPF vibration is observable with MCSA at

$$
f_{B P F}=-(1-2 s) f_{s},(3-2 s) f_{s} .
$$

Considering that rotor faults $(\mathrm{RF})$ in the rotor cage produce left sideband components in the current spectrum given by

$$
f_{R F}=\left(1-2 k_{2} s\right) f_{s},
$$

the $f_{B P F}$ and $f_{R F}$ frequencies are identical with opposite signs at the frequency shown in (6), if $k_{1} N_{b} / P$ is equal to 1 (for $k_{2}=1$ ).

$$
f_{R F}=-f_{B P F}=(1-2 s) f_{s} \quad\left(k_{2}=1\right)
$$

If the number of blades is not an integer multiple of the number of poles, more specifically if $k_{1} N_{b} / P$ is not equal to 1 , the BPF influence in MCSA is not in the vicinity of $-(1-2 s) f_{s}$ or $(1-2 s) f_{s_{2}}$ as can be seen in (3). Therefore, it does not interfere with MCSA based rotor fault detection. The rotor fault sideband frequency components are also produced on the right side of $f_{s}$; however, this component alone is not considered a reliable indicator of rotor faults since it depends heavily on the rotational inertia of the motor and load [27]. The right sideband component is not influenced by BPF vibrations, but cannot provide a consistent and reliable indication of rotor faults for medium-high voltage machines with large inertia.

From (6), it can be seen that a rotor fault produces a stator current vector, $\vec{l}_{S, R F}^{+}$, that rotates in the direction of rotor rotation (positive $(+)$ sequence) at $f_{R F}=(1-2 s) f_{s}$, and BPF vibration induces a vector, $\vec{l}_{S, B P F}^{-}$, that rotates in the opposite direction (negative (-) sequence) at the same frequency. The relative direction of rotation between the $\vec{l}_{S, R F}^{+}$and $\vec{\imath}_{s, B P F}^{-}$ vectors are illustrated in Fig. 2(a). The stator current phasors of the $(1-2 s) f_{s}$ component produced by a rotor fault (positive sequence) for each phase can be represented as,

$$
\begin{gathered}
\tilde{I}_{a s, R F}^{+}=I_{R F} \cos \left((1-2 s) \omega_{s} t\right), \\
\tilde{I}_{b s, R F}^{+}=I_{R F} \cos \left((1-2 s) \omega_{s} t-2 \pi / 3\right), \\
\tilde{I}_{c s, R F}^{+}=I_{R F} \cos \left((1-2 s) \omega_{s} t+2 \pi / 3\right),
\end{gathered}
$$

where $I_{R F}$ is the peak amplitude of the $f_{R F}$ component. The (1$2 s) f_{s}$ component produced by BPF vibration (negative sequence) can be expressed as,

$$
\begin{gathered}
\tilde{I}_{a s, B P F}^{-}=I_{B P F} \cos \left((1-2 s) \omega_{s} t+\alpha\right), \\
\tilde{I}_{b s, B P F}^{-}=I_{B P F} \cos \left((1-2 s) \omega_{s} t+2 \pi / 3+\alpha\right), \\
\tilde{I}_{c s, B P F}^{-}=I_{B P F} \cos \left((1-2 s) \omega_{s} t-2 \pi / 3+\alpha\right),
\end{gathered}
$$

where $\alpha$ is the relative phase angle between $\tilde{I}_{a s, R F}^{+}$and $\tilde{I}_{a s, B P F}^{-}$, and $I_{B P F}$ is the peak amplitude of the $f_{B P F}$ component. The individual rotor fault and BPF phasors and the relative phase angle $\alpha$ are illustrated in Fig. 2(b) for the case where $I_{R F}=I_{B P F}$.

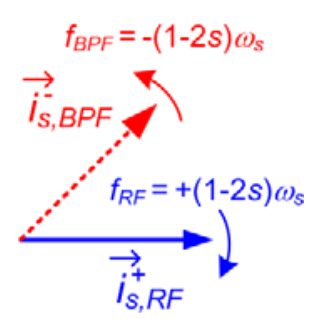

(a)

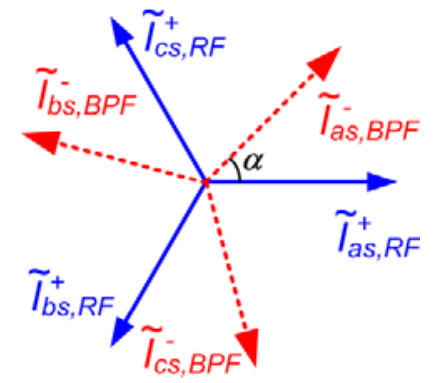

(b)
Fig. 2 (a) Space vector and (b) phasor representation of (1-2s) $f_{s}$ components induced in the stator current by rotor fault (RF) and blade pass frequency $(\mathrm{BPF})$ vibration (example for $I_{R F}=I_{B P F}$ ) 


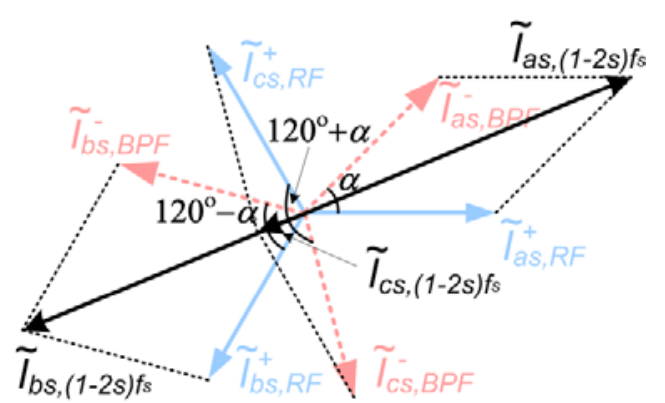

Fig. 3 Measurements of $(1-2 s) f_{s}$ components induced in individual phases a, $\mathrm{b}$, c of the stator current when rotor fault (RF) and blade pass frequency (BPF) vibration co-exist (example for case shown in Fig. 2)

If a rotor fault is present in the motor with high BPF vibration due to the load, $f_{R F}$ and $f_{B P F}$ components co-exist in the stator current. The $(1-2 s) f_{s}$ component of the stator current vector is the sum of the $\vec{l}_{S, R F}^{+}$and $\vec{l}_{S, B P F}^{-}$vectors under this condition, and therefore, measurements of the individual phase currents are the sum of the individual phase currents of the rotor fault and BPF components shown in (7)-(9) and (10)-(12), respectively. An example of the stator phase current measurements of the $(1-2 s) f_{s}$ components produced by a rotor fault and BPF vibration are illustrated in Fig. 3 for the case of Fig. 2. The two components, $I_{R F}$ and $I_{B P F}$, have different amplitudes in general, and the relative angle, $\alpha$, between them is random since it depends on 1) the relative angular position between the rotor fault and blade and 2) the load-dependent dynamics of the fluid flow in the pump, fan, or compressor. It can be shown that the peak amplitudes of phasors $\tilde{I}_{a s,(1-2 s) f_{s}}$, $\tilde{I}_{b s,(1-2 s) f_{s}}, \tilde{I}_{c s,(1-2 s) f_{s}}$ can be derived from (7)-(12), by adding the analytical equations of the components produced by rotor faults and BPF vibration for each individual phase as

$$
\begin{gathered}
\left|\tilde{I}_{a s,(1-2 s) f_{s}}\right|=\sqrt{I_{R F}^{2}+2 I_{R F} I_{B P F} \cos \alpha+I_{B P F}^{2}}, \\
\left|\tilde{I}_{b s,(1-2 s) f_{s}}\right|=\sqrt{I_{R F}^{2}+2 I_{R F} I_{B P F} \cos \left(\alpha-120^{\circ}\right)+I_{B P F}^{2}}, \\
\left|\tilde{I}_{c s,(1-2 s) f_{s}}\right|=\sqrt{I_{R F}^{2}+2 I_{R F} I_{B P F} \cos \left(\alpha+120^{\circ}\right)+I_{B P F}^{2}} .
\end{gathered}
$$

The equations derived in (13)-(15) show that the measurements of the phase a, $\mathrm{b}$, and c stator currents are a function of $I_{R F}, I_{B P F}$ and $\alpha$. The potential false rotor fault indications that can be produced with MCSA is presented in the following section based on (7)-(15) and Figs. 2-3.

\section{FALSE MCSA Rotor FAULT INDICATIONS PRODUCED BY BPF VIBRATION}

\section{A. False Positive Rotor Fault Indication due to BPF Vibration in Healthy Motor}

If the BPF vibration is large enough to induce a $-(1-2 s) f_{s}$ component in the stator current as shown in (3)-(4) for any reason listed in II.A., a false positive rotor fault alarm can be produced when applying MCSA to a motor with a healthy rotor cage. There are a number of MCSA products that rely on the measurement and analysis of the spectrum of one of the three phases of the stator current [1]-[2]. If measurement of only one phase is available, positive and negative sequence current vectors, $\vec{l}_{S, R F}^{+}$and $\vec{\imath}_{s, B P F}^{-}$, cannot be distinguished. In this case, the $f_{B P F}=-(1-2 s) f_{s}$ component is observed at $+(1-2 s) f_{s}$ and overlaps with $f_{R F}$ in the current spectrum producing a false positive rotor fault alarm. The commercially available MCSA products that measure all three phases monitor the $f_{R F}$ component of each individual phase current, or monitor the average of the $f_{R F}$ components obtained from the three phases [28]-[29]; therefore, false rotor fault alarms cannot be avoided. Since unnecessary inspection of the rotor due to a false alarm can be very costly, the maintenance engineer must be aware of the BPF induced false MCSA rotor fault alarms.

\section{B. False Rotor Fault Indications due to Co-existence of Rotor Fault and BPF Vibration}

If large BPF vibrations induce the $-(1-2 s) f_{s}$ component in a motor with a faulty rotor cage, and one phase of the current is analyzed, the two components, $f_{R F}$ and $f_{B P F}$, overlap at the same frequency. As a result of the interaction, the magnitude of the $(1-2 s) f_{s}$ components for phases $\mathrm{a}, \mathrm{b}$, and c are different, since they are sum of phasors consisting of $a-b-c$ and $a-c-b$ sequences, shown in Fig. 2(b) (the direction of the rotating vectors, $\vec{l}_{S, R F}^{+}$and $\vec{l}_{S, B P E}^{-}$, is opposite.). This can be observed in (13)-(15) and has been illustrated in Fig. 3 for clarity. The magnitude of $\tilde{I}_{a s,(1-2 s) f_{s}}$ as a function of the ratio between $I_{B P F}$ and $I_{R F}\left(I_{B P F} / I_{R F}\right)$ for different values of $\alpha$ are calculated from (13), and plotted in Fig. 4 for $I_{R F}=1$. It can be observed from this figure that the value of $\left|\tilde{I}_{a s,(1-2 s) f_{s}}\right|$ (and thus $\left|\tilde{I}_{b s,(1-2 s) f_{s}}\right|$, $\left.\left|\tilde{I}_{c S,(1-2 s) f_{s}}\right|\right)$ depends heavily on $I_{R F}, I_{B P F}$, and $\alpha$. The values of $\left|\tilde{I}_{a s,(1-2 s) f_{s}}\right|,\left|\tilde{I}_{b s,(1-2 s) f_{s}}\right|$, and $\left|\tilde{I}_{c s,(1-2 s) f_{s}}\right|$ as a function of $\alpha$ are calculated from (13)-(15), and plotted in Fig. 5 for the case of $I_{R F}=I_{B P F}=1$. It is shown in Fig. 5 that the magnitude of the (1-

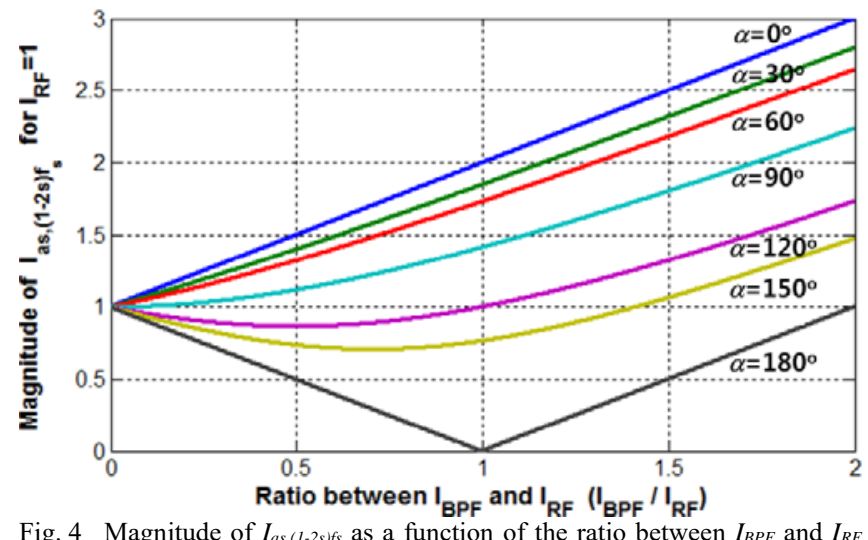

Fig. 4 Magnitude of $I_{a s,(1-2 s) f s}$ as a function of the ratio between $I_{B P F}$ and $I_{R F}$ $\left(I_{B P F} / I_{R F}\right)$ for different values of $\alpha$ for $I_{R F}=1$

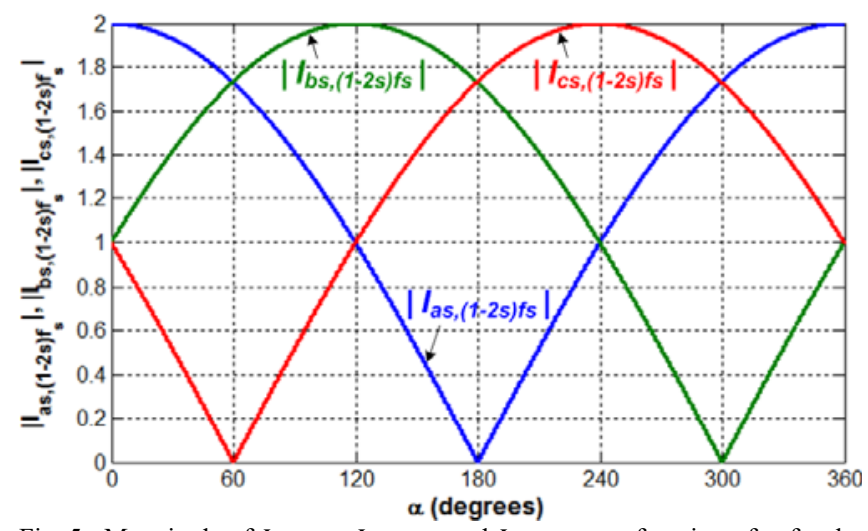

Fig. 5 Magnitude of $I_{a s,(1-2 s) f s}, I_{b s,(1-2 s) f s}$, and $I_{c s,(1-2 s) f s}$ as a function of $\alpha$ for the case of $I_{R F}=I_{B P F}=1$ 
$2 s) f_{s}$ components for each phase are completely different depending on $\alpha$, if rotor faults and BPF vibration co-exist. Some observations on how rotor faults and BPF vibration interact to produce false rotor fault indications with MCSA can be made from the analysis in II-III and Figs. 4-5, as summarized below.

- A false rotor fault alarm can be produced by $f_{B P F}$ for the case where $f_{R F}$ is small, if the $f_{R F}$ and $f_{B P F}$ components add in the phase in which MCSA is performed. A small $f_{R F}$ component due to the inherent asymmetry in the rotor cage that would not be regarded as a rotor fault could be amplified by the BPF vibration (false positive).

- MCSA can fail to detect the rotor fault, if the $f_{R F}$ and $f_{B P F}$ components cancel in the phase in which MCSA is performed. A $f_{R F}$ component large enough to trigger a rotor fault alarm could decrease to a small value below the fault threshold due to the $f_{B P F}$ component (false negative).

- The values of the (1-2s) $f_{s}$ components for phases $a, b$, and c are completely different, if $f_{R F}$ and $f_{B P F}$ components coexist, and $I_{R F}$ and $I_{B P F}$ cannot be separated. A false positive or negative indication can be produced depending on which phase(s) are being monitored.

The analysis provided in II-III clearly show that the influence of BPF vibration can produce false positive or negative rotor faults with the conventional MCSA based on measurement of one phase current. It can be seen that the influence of rotor fault and BPF vibration cannot be separated to avoid false alarms since the values of $I_{R F}, I_{B P F}, \alpha$ are unknown and random. Alternative methods for reliable detection of rotor faults under the BPF influence are presented in the following section based on the analysis provided in II-III.

\section{Reliable Detection of Rotor Faults Under BPF INFLUENCE}

The best way to avoid false MCSA indications due to BPF interference is to select a fan, pump, or compressor for which the number of motor poles is not an integer multiple of the number of blades. However, this cannot be done for the installed units since this is not a design or selection criterion in motor-pump systems. An alternative option for testing the rotor condition immune to $\mathrm{BPF}$ vibrations is to perform any type of assembled or disassembled off-line test. Off-line tests can provide reliable detection of rotor faults since they are not influenced by the load (and BPF vibration) because the motor is at standstill. There are many off-line tests available for rotor cage condition assessment that have been developed and verified over a long period of time [30]-[31]. One of the limitations of off-line testing is the requirement of motor disassembly for direct inspection/testing on the rotor surface. One option for testing the rotor without rotor disassembly is to perform the off-line single phase rotation test, where the current is monitored as the rotor is manually rotated with an ac voltage source connected between two phases [30]-[31]. This test is capable of reliable rotor testing independent of BPF since it is independent of load; however, the motor has to be stopped for testing, and manual rotation of the rotor shaft in discrete intervals is required, which is not feasible for many industrial applications.

\section{A. On-line Monitoring of Rotor Faults}

It was clearly shown in II-III that MCSA based on single phase current measurement can fail when the rotor fault and BPF induced components overlap since the positive $\left(f_{R F}\right)$ and negative $\left(f_{B P F}\right)$ sequence components of $(1-2 s) f_{s}$ cannot be separated. The easiest way of separating the positive and negative sequence components on-line is to observe the frequency spectrum of the current space vector, $\vec{l}_{s}$. The space vector $\vec{l}_{s}$ can be constructed if at least two phases of the current measurements are available from

$$
\vec{\imath}_{s}=\frac{2}{3} \cdot\left(i_{a s}+i_{b s} \cdot a+i_{c s} \cdot a^{2}\right),
$$

where $a$ is $e^{j \cdot 2 \pi / 3}$. The frequency spectrum of the complex number sequence $\vec{l}_{s}$ provides the full frequency spectrum between $-f_{\text {sam }} / 2$ and $+f_{\text {sam }} / 2$, for sampling frequency, $f_{\text {sam }}$. This allows separation of the positive and negative sequence components, which was not possible with the frequency spectrum of a single phase current $\left(i_{a s}, i_{b s}\right.$, or $\left.i_{c s}\right)$ that provides the spectrum between 0 and $f_{\text {sam }}$ with the positive and negative sequence components overlapped, as illustrated in Fig. 6. If the $\vec{l}_{S}$ spectrum is available, rotor faults can be detected by online monitoring of the $f_{R F}$ component, which can be obtained from the $(1-2 s) f_{s}$ component of the $\vec{l}_{s}$ spectrum, as shown in Fig. 6 (upper). This provides a reliable indication of rotor faults immune to BPF influence, since the $f_{B P F}$ component appears in the $-(1-2 s) f_{s}$ component of the $\vec{l}_{s}$ spectrum. It is also possible to monitor the presence of mechanical problems in the pump, fan, or compressor loads that result in excessive $\mathrm{BPF}$ vibrations by trending the $f_{B P F}$ component. This method can be easily implemented in any existing MCSA equipment if a minimum of two phase currents are measured.

\section{B. Monitoring of Rotor Faults under the Startup Transient}

Although spectrum analysis of the current space vector can provide a simple and effective solution, it can only be applied if steady state measurement of at least two phases of current is available. If only one of the three phase currents is measured, an option for detecting rotor faults independent of BPF vibrations is to analyze the startup current with time-frequency transformation techniques. Analysis of the stator current during startup is expected to provide reliable detection of rotor faults since BPF vibration is low when the rotor is accelerating

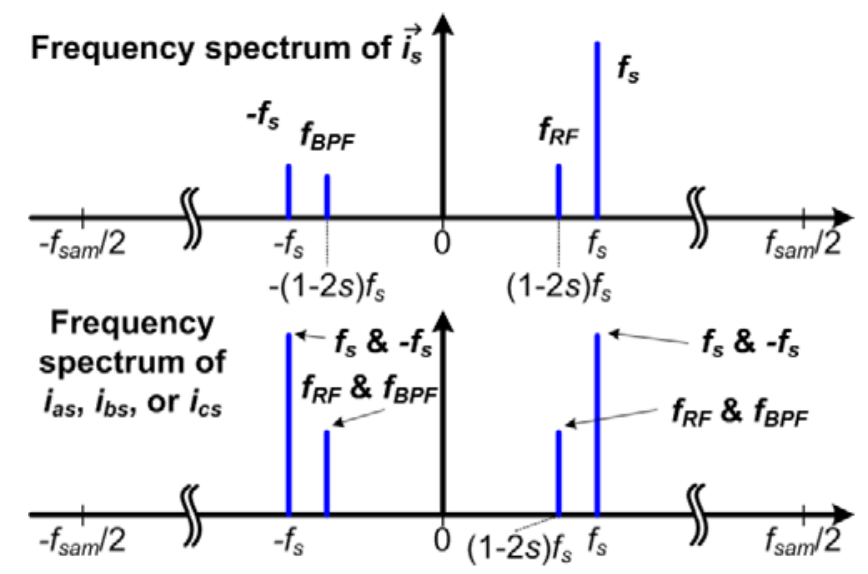

Fig. 6 Positive $\left(f_{s}, f_{R F}\right)$ and negative $\left(-f_{s}, f_{B P F}\right)$ sequence components in the frequency spectrum of the current space vector (upper), $\overrightarrow{\boldsymbol{i}}_{\boldsymbol{s}}$, and individual phase currents (lower), $i_{a s}, i_{b s}$, or $i_{c s}$. 
at low speed. There recently has been active research on timefrequency analysis of the startup current for rotor fault detection due to its performance and reliability benefits. The improved sensitivity of fault detection due to the large rotor current under startup, and its immunity to false indications have been reported in [6], [13], [20], [32]-[33].

When an induction motor is started directly from the line, the rotor slip decreases from 1 to the operating slip, which is close to 0 . As the slip decreases from 1 to 0 , it can be seen from (3) that the $f_{B P F}$ component induced in the stator current decreases from $60 \mathrm{~Hz}$ to a value close to $-60 \mathrm{~Hz}$ (if $k_{1} N_{b} / P=1$ ), and the $f_{R F}$ component shown in (5) increases from -60 to a value close to $60 \mathrm{~Hz}\left(k_{2}=1\right)$, as illustrated in Fig. 7. Since positive and negative frequency cannot be distinguished with measurement of one phase of the current, the evolution of $f_{B P F}$ and $f_{R F}$ is recognized as the absolute values, $\left|f_{B P F}\right|$ and $\left|f_{R F}\right|$. Therefore, they both decrease from $60 \mathrm{~Hz}$ to $0 \mathrm{~Hz}$ and increase to a value close to $60 \mathrm{~Hz}$ during the startup transient, as slip decreases from 1 to the operating slip $(\approx 0)$, as shown in Fig. 7 . Although the $f_{R F}$ and $f_{B P F}$ components have identical evolution under startup, the BPF component is negligible at low speed since the pressure applied to the impeller blade is low, whereas the rotor fault component is large due to the large rotor current.

There is a wide variety of time-frequency analysis methods that can be applied [32]-[33], but they would yield similar results since main idea is identical in that the evolution of the $f_{R F}$ component is observed over time during startup. In this work, the short time Fourier transform (STFT) is used since it provides a continuous 'picture' of the analyzed signal that shows its full time-frequency content, as shown in Fig. 8. The V-shaped evolution shown in Fig. 7 can be observed for a 6.6 $\mathrm{kV}, 3.8 \mathrm{MW}$ motor with a rotor fault (Fig. 8(a)), and the pattern disappears after rotor repair, as shown in Fig. 8(b). In this time-frequency map, the y-axis represents the frequency, and the $\mathrm{x}$-axis represents the time interval of the signal. The color at any point of the map at coordinates $\left[x_{0}, y_{0}\right]$ is related to the amplitude of the frequency component $y_{0}$ at the specific time $x_{0}$. An intense (bright) color in the map indicates that the frequency component has a strong presence in the analyzed signal at that specific instant of time. The main limitation of startup transient analysis is the requirement of motor startup with at least $1 \mathrm{sec}$. startup time, which could be difficult for applications that require continuous operation of the motor or motor applications with very low inertia.

\section{EXPERIMENTAL RESULTS}

\section{A. Experimental Test Setup}

An experimental study was performed on a custom-built centrifugal pump system to verify 1) the analysis on the influence of BPF vibrations on MCSA presented in II-III, and 2) the alternative test methods presented in IV for reliable rotor fault detection. A commercial $380 \mathrm{~V}, 2.2 \mathrm{~kW}, 2$ pole induction motor driving a centrifugal pump system, shown in Fig. 9(a), was configured to circulate the water through the tank. A pressure gauge was installed, and valves were used to adjust the load of the motor by controlling the water flow. To produce BPF vibrations that satisfy the $k_{1} N_{b} / P=1$ condition for interference with rotor fault detection, 2 of the 4 impeller blades were removed, as shown in Fig. 9(b). Healthy and faulty rotor cages were tested, where the faulty rotor cage was produced by drilling holes at the joint between the bar end ring.

A commercial data acquisition system, accelerometer (500 $\mathrm{mV} / \mathrm{g}$ ), and current transformers were used for vertical vibration, and stator current measurements. The steady state and startup current and vibration data were sampled at $6.4 \mathrm{kHz}$ (30 second interval for MCSA and vibration analysis). When obtaining the startup transient data, a high-inertia steel disc was

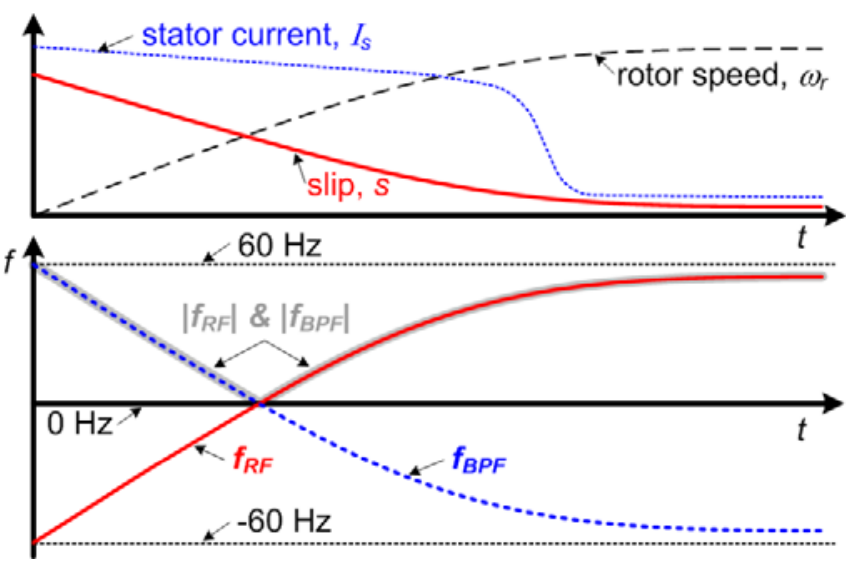

Fig. 7 Stator current, $I_{s}$, rotor speed, $\omega_{\mathrm{r}}$, and slip, $s$, waveforms under induction motor startup transient (upper); evolution of rotor fault, $f_{R F}$, and $\mathrm{BPF}$ vibration, $f_{B P F}$, components under startup transient

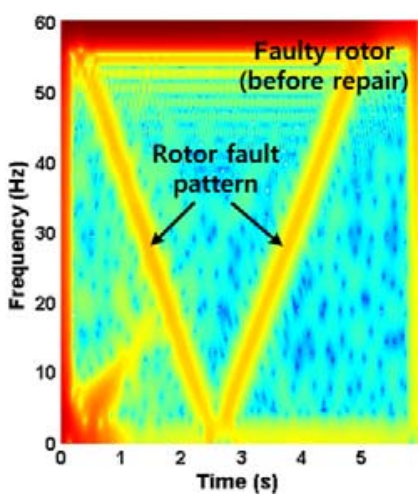

(a)

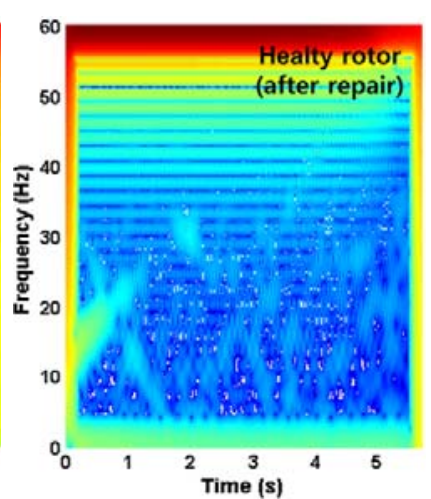

(b)
Fig. 8 STFT based startup transient analysis of $6.6 \mathrm{kV}, 3.8 \mathrm{MW}$ pump motor with a rotor fault (a) before and (b) after rotor fault repair

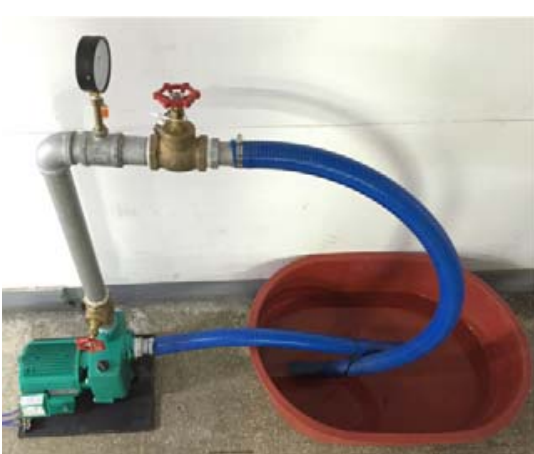

(a)

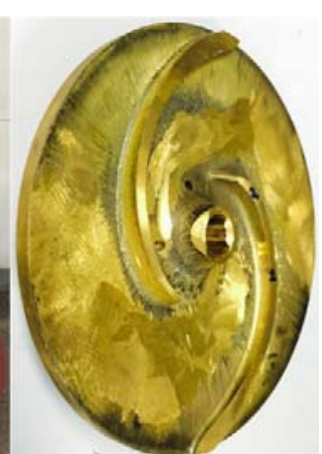

(b)
Fig. 9 (a) Motor-centrifugal pump test setup with water circulation through tank; (b) pump impeller with 2 blades 
installed on the shaft to increase the startup time to $1 \sim 1.5$ secs., which is typical for medium voltage motor pump applications.

\section{B. Experimental Results}

The valves were carefully adjusted while observing the amplitude of the BPF vibration to produce a large $f_{B P F}$ component in the stator current to verify the claims made in IIIII. Slow fluctuations in the amplitude of the BPF vibration and motor load were observed with the valve position fixed due to the dynamics of the water flow. A typical vibration measurement obtained with an accelerometer is shown in Fig. 10 for the system with a 2 blade impeller. It can be seen that integer multiples of the 2 times $f_{r}$ frequencies are noticeable due to $N_{b}=2$ blades, as predicted in (2). The $2 f_{r}(2 \mathrm{X})$ component is approximately 3 times larger than the rotor rotational frequency, $f_{r}(1 \mathrm{X})$, component shown in (1) due to the BPF influence. In most cases, the $f_{r}$ component is usually the largest due to the influence of misalignment, unbalance, eccentricity or other mechanical problems [23]-[24]. The integer multiples of $f_{r}$ in vibration are reflected in the stator current spectrum as sidebands of $f_{s}$ due to torque oscillations.

The results of MCSA performed on phases $a, b$ and $c$ are shown in Fig. 11(a)-(b) for a motor with healthy and faulty rotors, when BPF vibrations are present at $49 \%$ and $73.5 \%$ rated load, respectively. It can be seen that the amplitude of the $(1-2 s) f_{s}$ components are different for phases $a, b$, and $c$ due to the interaction between the positive and negative sequence components, $f_{R F}$ and $f_{B P F}$., as predicted in II-III. This confirms that $\mathrm{BPF}$ and rotor fault components produced at identical frequencies of $(1-2 s) f_{s}$ can add or cancel in phases a, b, and c depending on $\alpha$, if MCSA is performed on individual phases. Considering that the fault threshold for rotor faults is usually set between -50 and $-40 \mathrm{~dB}$ in the field, BPF vibration can produce a false positive alarm for a healthy rotor. The $(1-2 s) f_{s}$ component is $-48.8 \mathrm{~dB}$ for phase c in Fig. 11(a), which can be misinterpreted as a rotor fault although the rotor is in good condition. It can also be seen in the in Fig. 11(b) that a false negative indication can be produced for a faulty rotor if MCSA is performed on phase a $(-54.2 \mathrm{~dB})$, since the $f_{R F}$ and $f_{B P F}$ components cancel out in this phase. The amplitude of the right sideband, $(1+2 s) f_{s}$, components are the same for phases a, $\mathrm{b}$, and $\mathrm{c}$ for both cases shown in Fig. 11. This is because the right sideband is not influenced by BPF vibration, as they do not overlap. However, this component cannot be used as a reliable fault indicator for medium-high voltage motors since its magnitude depends heavily on the inertia of the motor and load [27].

The off-line single phase rotation test was performed on the motors with healthy and faulty rotors to verify the condition of the rotor. The stator current was measured in 10 degree intervals while a $47.5 \mathrm{~V}$ was applied across the terminals of phases $a$ and $b$. The results of the normalized stator current shown in Fig. 12 for healthy and faulty motors clearly indicate that the fault has been inserted properly. The results clearly show that the single phase rotation test can provide reliable detection of rotor fault independent of the BPF vibration, since it is a standstill test independent of load influence.

The positive and negative sequence spectra of the $\pm(1 \pm 2 s) f_{s}$ components obtained from the current space vector is shown for the healthy and faulty rotors in Figs. 13-14, respectively. The results show that the influence of BPF vibration and rotor fault can be separated since the BPF influence can be observed in the negative sequence, $-(1-2 s) f_{s}$, and the rotor fault in the positive sequence, $+(1-2 s) f_{s}$. For the motor with a healthy rotor,

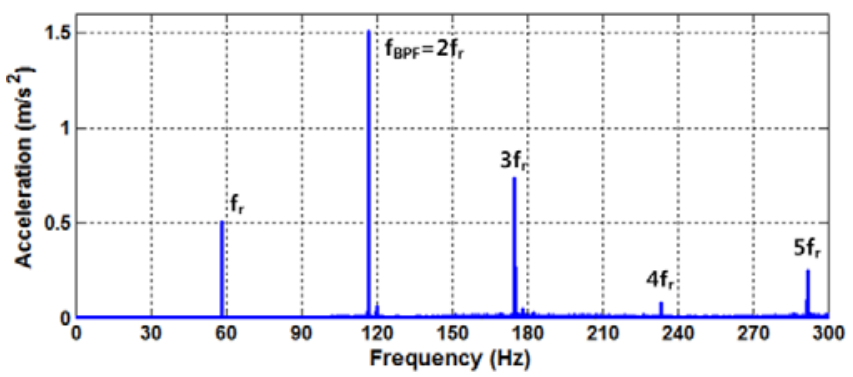

Fig. 10 Typical vertical vibration spectrum measured with an accelerometer under large BPF vibration with a 2 blade impeller (2 pole motor)

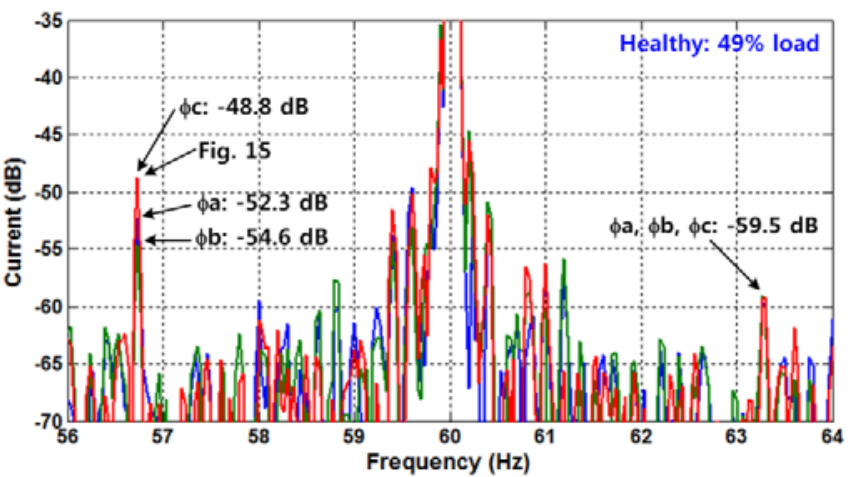

(a)

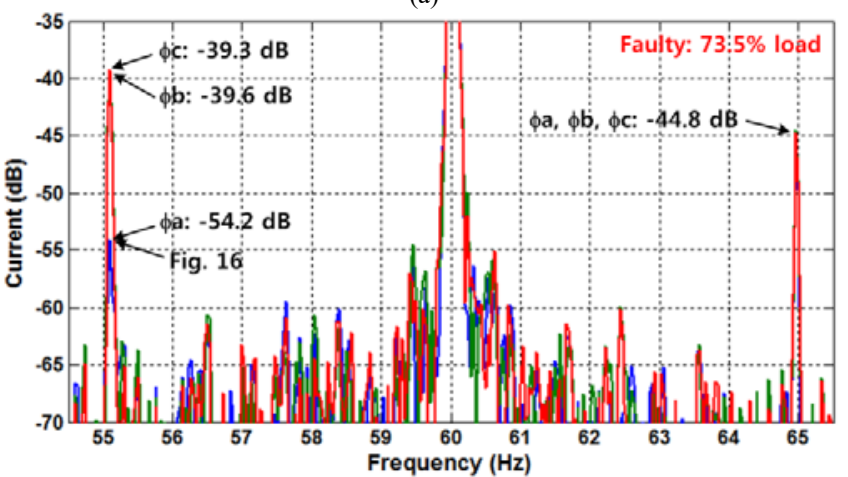

(b)

Fig. 11 MCSA results of $(1 \pm 2 s) f_{s}$ components for phase $a, b$, and c current of motor with (a) healthy (49\% rated load) and (b) faulty $(73.5 \%$ rated load) rotor with $\mathrm{BPF}$ interference

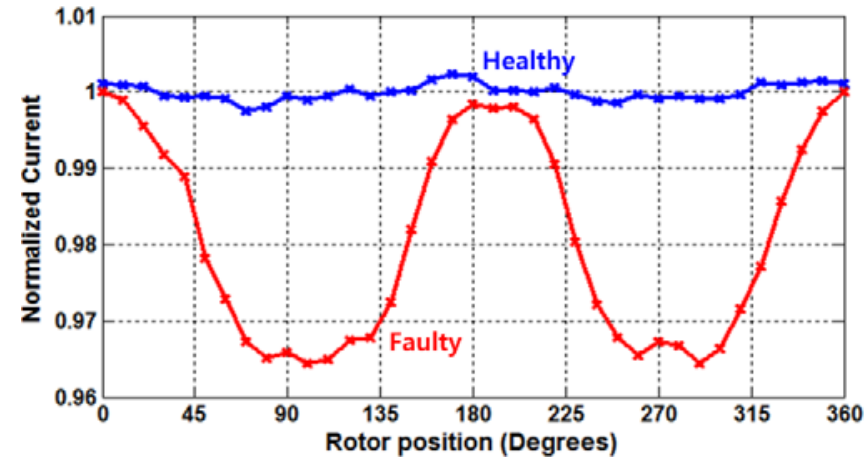

Fig. 12 Off-line standstill single phase rotation test results on motors with healthy and faulty rotors 
the amplitude of the $f_{R F}$ component is $-57.9 \mathrm{~dB}$ in Fig. 13(b), which indicates a healthy rotor, although the phase $\mathrm{c}$ measurement $(-48.8 \mathrm{~dB})$ shown in Fig. 11(a) indicated of a faulty rotor. The interaction between the $f_{R F}$ component (-57.9 $\mathrm{dB})$ and large $f_{B P F}$ component $(-52.2 \mathrm{~dB})$ shown in Fig. 13(a) caused the increase of the phase c component to $-48.8 \mathrm{~dB}$. For the faulty motor shown in Fig. 14, the $f_{R F}$ component at -43.1 $\mathrm{dB}$ in Fig. 14(b) indicates a rotor fault although the phase a current $(-54.2 \mathrm{~dB})$ in Fig. 11(b) indicates a healthy rotor. The $f_{B P F}$ component shown in Fig. 14(a) caused the decrease in the phase a current since the two components were out of phase. The influence of the rotor fault and BPF vibration can be clearly distinguished since the $f_{R F}$ component in the positive sequence spectrum (Figs 13(b), 14(b)) provides a reliable indication of a rotor fault. The $f_{B P F}$ component in the negative sequence spectrum (Figs. 13(a), 14(a)) can be used to monitor potential mechanical faults that cause excessive BPF vibrations. This is the easiest solution for reliable on-line detection of rotor faults independent of BPF vibration, if current measurements of two phases are available.

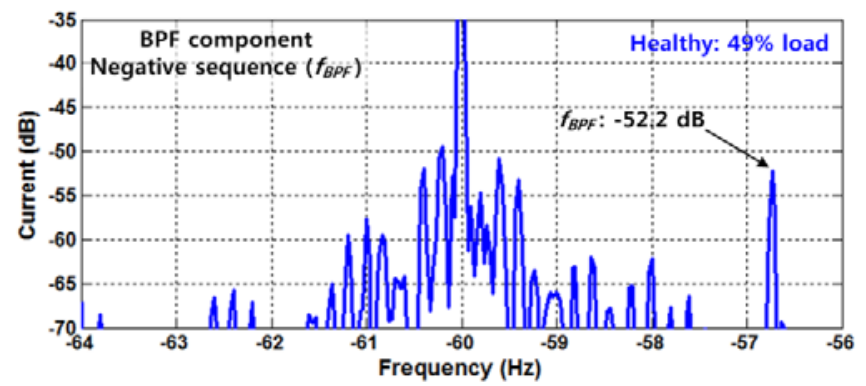

(a)

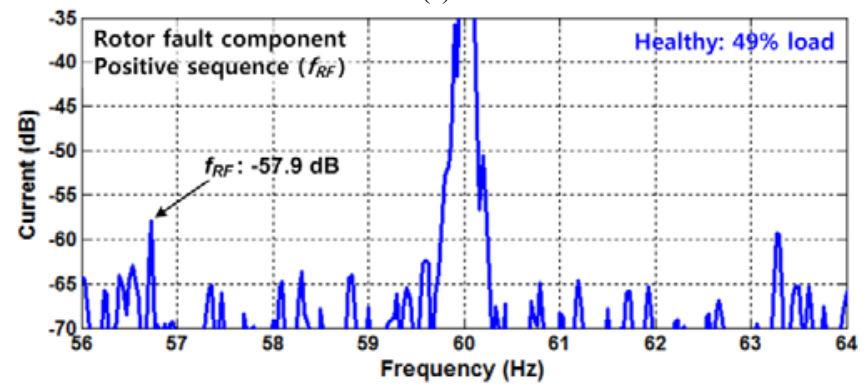

(b)

Fig. 13 MCSA results of current space vector for healthy rotor with BPF interference; (a) negative $\left(f_{B P F}\right)$ and (b) positive $\left(f_{R F}\right)$ sequence

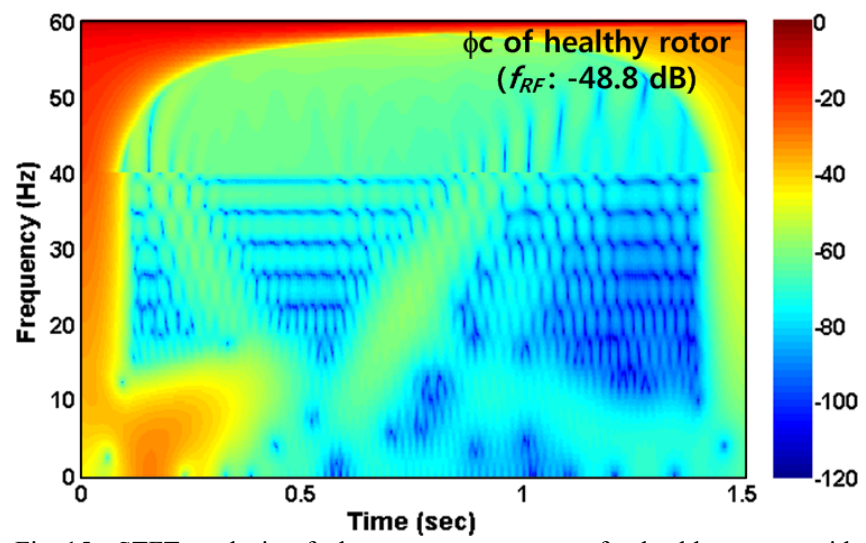

Fig. 15 STFT analysis of phase c startup current for healthy motor with BPF vibration (MCSA produced false positive alarm at $-48.8 \mathrm{~dB}$ )
The startup current measured from the three phases of the healthy and faulty motors with BPF vibration were analyzed with STFT. The results for phase $\mathrm{c}$ of the healthy motor that produced a false positive alarm $(-48.8 \mathrm{~dB})$, and phase a of the faulty motor that produced a false negative indication $(-54.2$ dB) are shown in Fig. 15-16, respectively. It can be seen that the evolution of the $f_{R F}$ pattern shown in Figs. 7-8 can only be observed for the faulty motor in Fig. 16 although the $(1-2 s) f_{s}$ component is smaller than that of the healthy motor in steady state (Fig. 11). The startup test results are identical in the other phases since BPF vibration is negligible at low speed during startup, and only the influence of the rotor fault is reflected. The results of Figs. 13-16 clearly show that MCSA of the current space vector and startup analysis provide reliable detection of rotor faults independent of BPF vibration for cases where MCSA based on one phase current fails.

\section{CONCLUSION}

The influence of BPF vibration in pumps, fans, and compressors on MCSA based rotor fault detection of induction

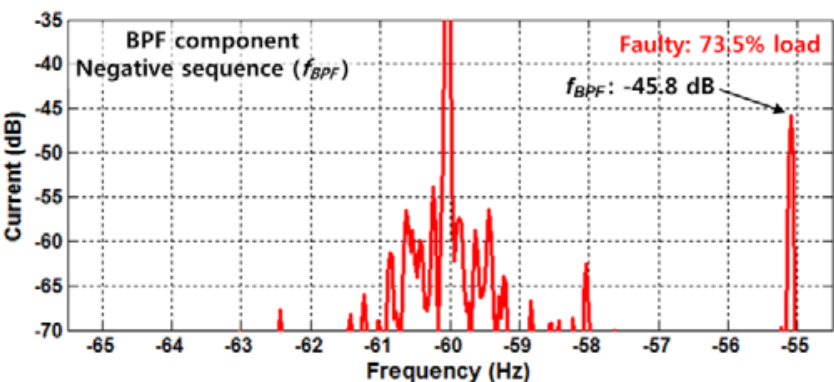

(a)

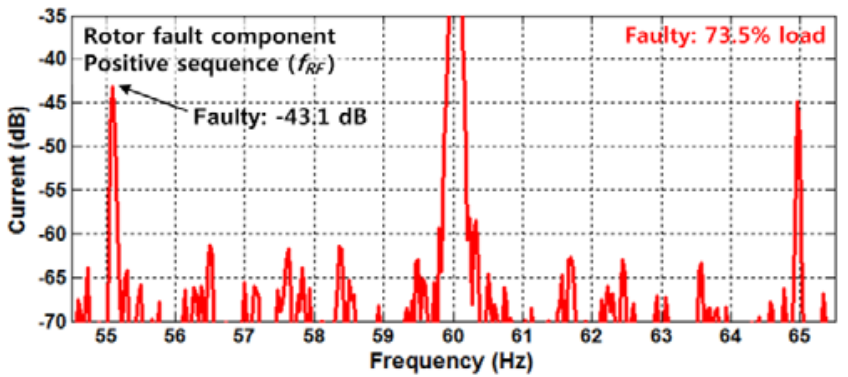

(b)

Fig. 14 MCSA results of current space vector for faulty rotor with BPF interference; (a) negative $\left(f_{B P F}\right)$ and (b) positive $\left(f_{R F}\right)$ sequence

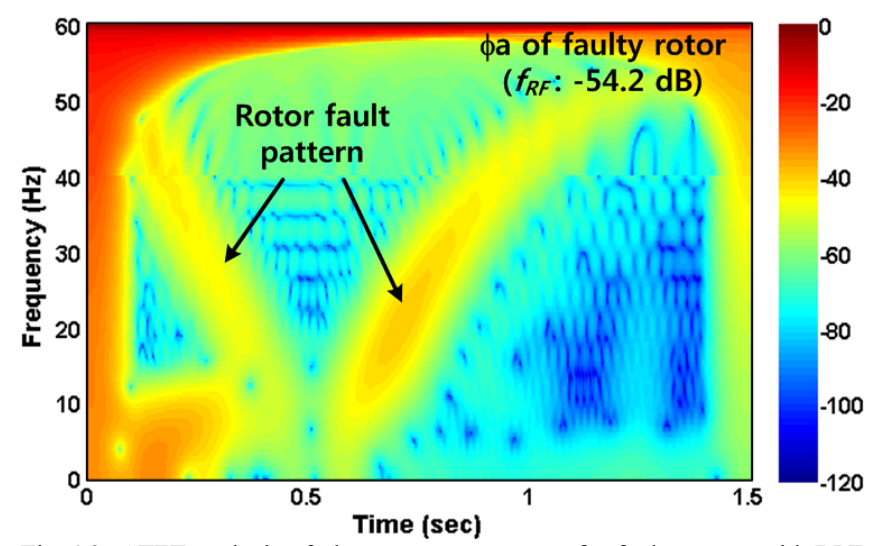

Fig. 16 STFT analysis of phase a startup current for faulty motor with BPF vibration (MCSA produced false negative alarm at $-54.2 \mathrm{~dB}$ ) 
machines was evaluated in this paper. A theoretical analysis shows that the influence of BPF vibrations can interfere with the rotor fault frequency components, if the number of motor poles is an integer multiple of the number of blades. It is shown for the first time in this work that BPF vibrations can produce false positive or negative rotor fault indications causing conventional MCSA based on one phase current to fail. It is shown that analysis of the positive sequence component of the current space vector spectrum can provide a simple solution for reliable on-line detection of rotor faults immune to BPF interference. It is also shown that all off-line tests and startup transient analysis can provide reliable detection of rotor faults independent of BPF influence. The potential of monitoring mechanical defects that give rise to BPF vibration has also been shown. All claims made in the paper are verified experimentally on an induction motor-centrifugal pump system setup. Considering that a significant percentage of mediumhigh voltage induction motors are used for driving loads with BPF vibration, the findings of this paper are expected to help prevent false MCSA indications in the field.

\section{ACKNOWELDGMENT}

The authors would like to thank Hansung Heavy Industries CO. LTD. for sharing their experience on design and operation of pumps driven by medium-high voltage induction motors.

\section{REFERENCES}

[1] W.T. Thomson, M. Fenger, "Current signature analysis to detect induction motor faults," IEEE Ind. Appl. Mag., vol. 7, no. 4, pp. 26-34, July/Aug. 2001.

[2] I.M. Culbert, W. Rhodes, "Using current signature analysis technology to reliably detect cage winding defects in squirrel-cage induction motors," IEEE Trans. on Ind. Appl., vol. 43, no.2, pp.422-428, Mar./Apr. 2007.

[3] W.T. Thomson, "On-line current monitoring - the influence of mechanical loads or a unique rotor design on the diagnosis of broken rotor bars in induction motors," Proc. of ICEM, pp. 1236-1240, 1992.

[4] D. Leith, D. Rankin, "Real time expert system for identifying rotor faults and mechanical influences in induction motor phase current," Proc. of IEE Electric Machines and Drives, Sept. 1991.

[5] G.B. Kliman, R.A. Koegl, J. Stein, R.D. Endicott, M.W. Madden, "Noninvasive detection of broken rotor bars in operating induction motors," IEEE Trans. on Ener. Conv., vol. 3, no. 4, pp. 873-879, Dec 1988.

[6] S.B. Lee, et. al. "Identification of false rotor fault indications produced by on-line MCSA for medium voltage induction machines," IEEE Trans. on Ind. Appl., vol. 52, no. 1, pp. 729-738, Jan./Feb. 2016.

[7] A. Bellini, et al., "On-field experience with on-line diagnosis of large induction motors cage failures using MCSA," IEEE Trans. on Ind. Appl., pp. 1045-1053, vol. 38, no. 4, July/Aug. 2002.

[8] S. Lee, J. Hong, S.B. Lee, E. Wiedenbrug, M. Teska, H. Kim, "Evaluation of the Influence of Rotor Axial Air Duct Design on Condition Monitoring of Induction Motors," IEEE Trans. on Ind. Appl., vol. 49, no. 5, Sept./Oct. 2013.

[9] B.D. Evans, "Induction motor case histories: a focus on electrically related phenomena," Proc. of Vibration Institute Annual Meeting, 2009.

[10] S. Shin, J. Kim, S.B. Lee, C. Lim, E.J. Wiedenbrug, "Evaluation of the Influence of Rotor Magnetic Anisotropy on Condition Monitoring of 2 Pole Induction Motors," IEEE Trans. on Ind. Appl., vol. 51, no. 4, pp. 2896-2904, Jul./Aug. 2015.

[11] T.M. Wolbank, J.L. Machl, P. Macheiner, H. Hauser, "Extraction and elimination of induction machines inherent asymmetry caused by lamination material anisotropy," Proc. of Pwr Electr. and Drive Syst., vol. 1, pp. 508-513, Nov. 2003.
[12] S. Williamson, M.A.S. Abdel-Magied, "Steady state analysis of double cage induction motors with rotor cage faults," IEE Proc. - Elec. Pwr. Appl., vol. 134, no. 4, pp. 199-206, July 1987.

[13] J. Antonino-Daviu, M. Riera-Guasp, J. Pons-Llinares, J. Park, S.B. Lee, J. Yoo and C. Kral, "Detection of Broken Outer-Cage Bars for DoubleCage Induction Motors under the Startup Transient," IEEE Trans. on Ind. Appl., vol. 48, no. 5, pp. 1539-1548, Sept./Oct. 2012.

[14] G.Y. Sizov, A. Sayed-Ahmed, C. Yeh, N.A.O. Demerdash, “Analysis and diagnostics of adjacent and nonadjacent broken-rotor-bar faults in squirrel-cage induction machines," IEEE Trans. on Ind. Electr., vol. 56, no. 11, pp. 4627-4641, Nov. 2009.

[15] T.J. Sobczyk, W. Maciolek, "Diagnostics of rotor-cage faults supported by effects due to higher MMF harmonics," Proc. of Power Tech. Conf., vol. 2, pp. 5-9, June 2003.

[16] M. Riera-Guasp, M.F. Cabanas, J.A. Antonino-Daviu, M. PinedaSanchez, C.H.R. Garcia, "Influence of nonconsecutive bar breakages in motor current signature analysis for the diagnosis of rotor faults in induction motors," IEEE Trans. on Ener. Conv., vol. 25, no. 1, pp. 80-89, March 2010.

[17] G.R. Bossio, C.H. De Angelo, J.M. Bossio, C.M. Pezzani, G.O. Garcia, "Separating broken rotor bars and load oscillations on IM fault diagnosis through the instantaneous active and reactive currents," IEEE Trans. on Ind. Electr., vol. 56, no. 11, pp. 4571-4580, Nov. 2009.

[18] S.M.A. Cruz, "An active-reactive power method for the diagnosis of rotor faults in three-phase induction motors operating under timevarying load conditions," IEEE Trans. on Ener. Conv., vol. 27, no. 1, pp. 71-84, March 2012.

[19] M. Drif, A.J.M. Cardoso, "Discriminating the simultaneous occurrence of three-phase induction motor rotor faults and mechanical load oscillations by the instantaneous active and reactive power media signature analyses," IEEE Trans. on Ind. Electr., vol. 59, no. 3, pp. 1630-1639, March 2012.

[20] H. Kim, S.B. Lee, S.B. Park, S.H. Kia, G.A. Capolino, "Reliable detection of rotor faults under the influence of low frequency load torque oscillations for applications with speed reduction couplings," IEEE Trans. on Ind. Appl., vol. 52, no.2, pp. 1460-1468, Mar./Apr. 2016.

[21] Office of Energy Efficiency and Renewable Energy, US DOE, "United States Industrial Electric Motor Systems Market Opportunities Assessment," Dec. 2002.

[22] I.J. Karassik, T. McGuire, Centrifugal pumps, Chapman \& Hall, 1998.

[23] V. Wowk, Machinery vibration - measurements and analysis, McGraw Hill, 1991.

[24] J.E. Berry, "Analysis III: introduction to special vibration diagnostic techniques and how to analysis low, high, and variable speed machines," Technical Associates of Charlotte, 1998.

[25] M. Blodt, P. Granjon, B. Raison, G. Rostaing, "Models for bearing damage detection in induction motors using stator current monitoring," IEEE Trans. on Ind. Electr., vol. 55, no. 4, pp. 1813-1822, April 2008.

[26] F. Immovilli, A. Bellini, R. Rubini, C. Tassoni, "Diagnosis of bearing faults in induction machines by vibration or current signals: a critical comparison," IEEE Trans. on Ind. Appl., vol. 46, no. 4, pp. 1350-1359, July/Aug. 2010.

[27] F. Filippetti, G. Franceschini, C. Tassoni, P. Vas, "AI techniques in induction machines diagnosis including the speed ripple effect," IEEE Transactions on Industry Applications, vol. 34, no. 1, pp. 98-108, Jan./Feb. 1998.

[28] Baker Instrument Company, an SKF Group Company, SKF Dynamic Motor Analyzer: EXP4000 User manual, Fort Collins, CO, 2012.

[29] D.L. Mckinnon, "Fault zone analysis - identifying motor defects using the rotor fault zone," PDMA Corporation, Tampa, FL, 2011 [Online]. Available: http://www.pdma.com/pdfs/Articles/

[30] T. Bishop, "Squirrel cage rotor testing," EASA Convention, 2003.

[31] D. Hyun, S. Lee, J. Hong, S.B. Lee, S. Nandi, "Detection of Airgap Eccentricity for Induction Motors using the Single-Phase Rotation Test," IEEE Trans. on Ener. Conv., vol. 27, no. 3, pp. 689-696, Sept. 2012.

[32] S. Mallat, A Wavelet Tour on Signal Proc., Academic Press, NY, 1999.

[33] Z. Feng, M. Liang, F. Chu, "Recent advances in time-frequency analysis methods for machinery fault diagnosis: a review with 
